\title{
Vorarlberg in den Krisenjahren 1816 und 1817: Gründe und Auswirkungen
}

\section{Florian Guggenberger}

Kerngebiet: Wirtschafts- und Sozialgeschichte

eingereicht bei: Univ.-Prof. Dr. Patrick Kupper und Reinhard Nießner, MA

eingereicht im: WiSe 2019/20

Rubrik: Seminar-Arbeit

\begin{abstract}
Vorarlberg in the Crisis Years 1816 and 1817: Reasons and Effects

Between 1812 and 1817, the so-called "Little Ice Age" in Central Europe reached its climax. During this period, the summers were two to three degrees colder than the 20th century average. In addition to the economic depression caused by the Napoleonic Wars, the eruption of the volcano Tambora in April 1815 further exacerbated the situation. The consequences of the climatic changes affected the whole world and resulted in the "Year Without a Summer" in 1816. The effects of the climatic fluctuation also had an impact on the following year with famines and natural disasters still being a huge problem. Also in Vorarlberg, this resulted in terrible disasters: Flooding, avalanches and crop failures led to years of crisis in Vorarlberg.
\end{abstract}

\section{Einleitung}

Zwischen 1812 und 1817 erreichte die sogenannte "Kleine Eiszeit" in Mitteleuropa ihren Höhepunkt. In dieser Zeit waren die Sommer zwei bis drei Grad kälter als im Durchschnitt des 20. Jahrhunderts.' Zusätzlich zu der schon herrschenden Depression aufgrund der Napoleonischen Kriege wurde die Situation durch den Ausbruch des Vulkans Tambora in Indonesien im April 1815 verschärft. Dessen Folgen waren weltweit spürbar und führten 1816 zum sogenannten „Jahr ohne Sommer“. Auch 1817 konnten

Christian Pfister, Klimageschichte der Schweiz 1525-1860. Das Klima der Schweiz von 1525-1860 und seine Bedeutung in der Geschichte von Bevölkerung und Landwirtschaft, Bd. 1 (Academica helvetica 6), Bern-Stuttgart 21985, S. 131. 
die Auswirkungen des kälteren Klimas und des Ausbruchs durch Hungerkrisen und Naturkatastrophen von den Zeitgenoss*innen noch wahrgenommen werden.

Im Zuge dieser Seminararbeit wird die Lage in Vorarlberg während jener Krisenjahre näher betrachtet. Die Analyse dieser Thematik soll entlang folgender Fragestellungen durchgeführt werden: War der Ausbruch des Vulkans Tambora der Hauptgrund für das "Jahr ohne Sommer" 1816? Wie wirkte sich die (Klima-)Krise der Jahre 1816 und 1817 auf Vorarlberg aus? Mit welchen Auswirkungen und Problemen war die Vorarlberger Bevölkerung konfrontiert?

Anknüpfend an diese Forschungsfragen lautet die erste These dieser Arbeit, dass der Ausbruch des Tambora nicht als einziger Grund für das „Jahr ohne Sommer" und das nachfolgende Krisenjahr 1817 angesehen werden kann, sondern eine Vielzahl von Faktoren und Entwicklungen, die teilweise (lange) vor dem Ausbruch einsetzten, miteinbezogen werden müssen. Von einer "Tamborakrise“2, wie Wolfgang Behringer es nennt, die hauptverantwortlich für das "Jahr ohne Sommer" gewesen sein soll und zudem historische Entwicklungen des 19. Jahrhunderts weitreichend beeinflusste, kann nicht die Rede sein. Die zweite These ergibt sich aus der ersten und besagt, dass der Ausbruch $1815 \mathrm{im}$ historischen Sinne als Auslöser für die Klimaanomalien in den beiden Folgejahren gesehen werden soll, der die bereits einsetzenden klimatischen Schwankungen sowie gesellschaftliche und wirtschaftliche Krisen verstärkte.

Im ersten Teil dieser Arbeit soll zunächst auf die Ursachen der Kälteperiode der 1810erJahre und der Krisensituation in Europa eingegangen werden, um so ein Bild der damaligen Rahmenbedingungen zu generieren. Darauf aufbauend wird der Ausbruch des Vulkans Tambora als einer der wesentlichen Faktoren für die Verschlechterung der damaligen klimatischen und sozioökonomischen Situation beschrieben. Im Fokus werden vor allem die unmittelbaren Folgen des Ausbruchs stehen. Als wichtigste Literatur dieses ersten Teils dient zum einen Wolfgang Behringers Monografie "Tambora und das Jahr ohne Sommer", in der sich Behringer speziell mit den gesellschaftlichen Auswirkungen des Tambora-Ausbruchs beschäftigte. ${ }^{3}$ Zum anderen ist aus klimageschichtlicher Perspektive der erste Band von Christian Pfisters Monografie über die "Klimageschichte der Schweiz 1525-1860" zu nennen, dessen klima- und agrargeschichtliche Untersuchungen für Vorarlberg ebenfalls relevant sind. ${ }^{4}$

Der Hauptteil der vorliegenden Arbeit untersucht die Situation in Vorarlberg in den Krisenjahren 1816 und 1817 genauer. Dabei soll herausgearbeitet werden, wie sich durch unterschiedlichste Faktoren die Lebenssituation der Vorarlberger Bevölkerung in diesen Jahren drastisch verschlechterte. Ausgelöst durch das schlechte Klima kam es zu Naturkatastrophen und untypischen Wettersituationen, die zu Ernteausfällen und erhöhten Getreidepreisen führten, was wiederum in einer extremen Hungerkrise und Mangelernährung der Bevölkerung resultierte. Als konkrete Beispiele für die Naturkata-

2 Wolfgang Behringer, Tambora und das Jahr ohne Sommer. Wie ein Vulkan die Welt in die Krise stürzte, München ${ }^{3} 2016$, S. 11.

3 Ebd

4 Pfister, Klimageschichte der Schweiz. 
strophen dieser Zeit sind zum einen das Bodenseehochwasser von 1817, das als bisher höchstes Hochwasser am Bodensee gilt, und zum anderen Lawinenabgänge in höher gelegenen Gebieten Vorarlbergs zu nennen. Auf die politische Ebene in Vorarlberg wird im Zuge dieser Arbeit nicht eingegangen, die klimatischen Rahmenbedingungen und Faktoren stehen im Zentrum der Untersuchung.

Als Forschungsstand zu diesen Krisenjahren in Vorarlberg soll die Monografie der Vorarlberger Historikerin Sabine Sutterlütti „JJammer, Elend und Noth!!' Vorarlberg 1814 bis 1818" angeführt werden, die als Erste eine umfangreiche Arbeit mit zeitgenössischen Quellen zu dieser Krisenzeit abfasste..$^{5}$ Michael Kasper schrieb in einem unter anderem von ihm selbst herausgegebenen Sammelband einen Beitrag mit dem Titel "Achtzehnhundertundzutodegefroren", in dem er sich speziell mit den Krisen und Ereignissen im südlichen Teil Vorarlbergs beschäftigte. ${ }^{6}$ Als historisches Überblickswerk zu Vorarlberg im behandelten Zeitraum ist Alois Niederstätters zweiter Band zur Geschichte Vorarlbergs zu nennen.7 Gerald Müllers Monografie „Hunger in Bayern 1816-1818“ bezieht sich auf dieselbe Situation in der Nachbarregion Bayern. ${ }^{8}$ Insgesamt muss der Forschungsstand zur Klimageschichte Vorarlbergs als noch sehr lückenhaft beschrieben werden. Sutterlüttis Arbeit ist bisher die einzige, die sich mit den Krisenjahren 1816/1817 aus klimageschichtlicher Perspektive beschäftigt. Auch historische Hochwasser auf der vorarlbergischen Seite des Bodensees sind bisher nicht untersucht. Die vorliegende Arbeit versteht sich dementsprechend als Beitrag zur Klimageschichte Vorarlbergs.

\section{Klima und Gesellschaft: Ursachen für die Krise(n) um 1815}

Klima und Gesellschaft sind zwei Begriffe, die oft in einem Atemzug genannt werden. So war es auch kein Zufall, dass (unter anderem) die Kälteperiode der 1810er-Jahre - der Höhepunkt der "Kleinen Eiszeit" - und der Ausbruch des Vulkans Tambora zu gesellschaftlichen Krisenerscheinungen auf der ganzen Welt führten. Das Zusammenspiel von diesen und weiteren Faktoren (ozeanische Variabilität, geringere Sonnenaktivität) brachte bereits in den Jahren vor dem Ausbruch des Tambora eine weitere globale Abkühlung. ${ }^{9}$ Gepaart mit einer wirtschaftlichen Depression in großen Teilen Europas nach den Napoleonischen Kriegen entstand als Folge eine extreme Krisensituation, die im "Jahr ohne Sommer" 1816 und dem nachfolgenden „Hungerjahr" 1817 ihren Höhenpunkt fand.

5 Sabine Sutterlütti, „Jammer, Elend und Noth!!”. Vorarlberger 1814 bis 1818 (Institut für sozialwissenschaftliche Regionalforschung Veröffentlichungen 14), Regensburg 2016.

6 Michael Kasper, „Achtzehnhundertundzutodegefroren“, in: Edith Hessenberger/Michael Kasper/Andreas Rudigier/ Bruno Winkler (Hrsg.), Jahre der Heimsuchung. Historische Erzählbilder von Zerstörung und Not im Montafon (Sonderband zur Montafoner Schriftenreihe 12), Schruns 2010, S. 9-71.

$7 \quad$ Alois Niederstätter, Geschichte Vorarlbergs, Bd. 2: Vorarlberg 1523 bis 1861. Auf dem Weg zum Land, Innsbruck 2015.

8 Gerald Müller, Hunger in Bayern 1816-1818. Politik und Gesellschaft in einer Staatskrise des frühen 19. Jahrhunderts (Europäische Hochschulschriften Reihe III, Geschichte und ihre Hilfswissenschaften 812), Frankfurt am Main u. a. 1998.

9 Stefan Brönnimann/Daniel Krämer, Tambora und das „Jahr ohne Sommer“ 1816. Klima, Mensch und Gesellschaft (Geographica Bernensia G90), Bern 2016, S. 28. 


\subsection{Kälteperiode und Nachkriegsdepression in Europa}

Die Krisenjahre 1816 und 1817 werden in der Forschung auf die klimatischen Gegebenheiten dieser Zeit zurückgeführt. Klimaforscher*innen bezeichnen wegen der tiefen Temperaturen die Zeit von etwa 1500 bis Mitte des 19. Jahrhunderts als „Kleine Eiszeit". In Mitteleuropa erreichte sie ihren Höhepunkt zwischen 1812 und 1817. In Vorarlberg gilt der Zeitraum von 1810 bis 1822 als letzte Phase dieser Kälteperiode. ${ }^{10}$ Der Schweizer Klima- und Umwelthistoriker Christian Pfister diagnostiziert für die Schweiz sogar eine Kaltperiode von 1812 bis 1860, in der die Jahrestemperatur zum Teil bis zu 0,6 Grad unter dem Durchschnittswert des 20. Jahrhunderts blieb. Pfister sieht aufgrund der "Dauer und Größe des thermischen Defizits [...] die ausgeprägteste Kaltperiode seit 1520"11. Er hebt vor allem das Jahrzehnt von 1810 bis 1819 hervor, das sich durch ein sommerliches Wärmemanko hervortat. Charakteristisch waren für diese Periode unfreundliche Sommer, die im Gebirge große Schneemengen brachten und mit einer Häufung von schweren Überschwemmungen einhergingen. ${ }^{12}$

Allen voran blieben die Sommer 1813 und 1816 um mehr als zwei Grad unter dem Durchschnitt des 20. Jahrhunderts. Auch die Sommer 1815 und 1821 waren über 1,5 Grad kälter, lediglich die Sommer 1818 und 1819 lagen im Durchschnitt des 20. Jahrhunderts. Pfister argumentiert weiter, dass die Jahre 1812 bis 1817 eindeutig die Züge einer Klimaschwankung tragen. Dem überdurchschnittlich sonnigen und warmen Jahr 1811 folgte 1812 ein Kältejahr, welches zu Überflutungen während aller Jahreszeiten führte. Von 1812 bis 1814 herrschten trockene Winter. Ab 1815 mündeten schneereiche Winter in späte Frühlinge. Die Sommer von 1812 bis 1816 bezeichnet Pfister als „Eiszeitsommer", die alle in feuchte und frostige Herbste übergingen. Im Alpengebiet verzögerte sich im Frühjahr die Schneeschmelze und im Herbst winterte es verfrüht ein. Konkrete ökologische Konsequenzen dieser Kältewirkung waren unter anderem schlecht ausfallende Weinernten und den Sommer überdauernde Schneedecken in Höhen von 1.800 bis 2.300 Metern. Dies führte teilweise zur Vernichtung der Rasen und zur langfristigen Schrumpfung der Tragfähigkeit von Weidegebieten, was katastrophale Auswirkungen auf die Viehhaltung hatte. Es kam aber auch zur Abschmelzung von riesigen Schneemengen, wodurch Seen und Flüsse im Voralpengebiet einen Hochstand erreichten, der seit 1566 nicht mehr erzielt worden war. ${ }^{13}$

Gründe für diese Klimaschwankungen müssen zum einen in der längerfristig reduzierten Sonnenaktivität in der Zeit des sogenannten "Dalton-Minimums" (etwa 1790 bis 1830) gesehen werden. Zum anderen lag es teilweise an den „Umstellungen der allgemeinen Zirkulation der Atmosphäre, die mit der verstärkten Zufuhr kühlerer Luftmassen aus Nordosteuropa und einem eher schwach entwickelten Azorenhoch in den Sommermonaten zusammenhingen"14. Verstärkt wurden diese Entwicklungen durch

10 Sutterlütti, ,Jammer, Elend und Noth!!", S. 53.

11 Pfister, Klimageschichte der Schweiz, S. 131.

12 Ebd.

13 Pfister, Klimageschichte der Schweiz, S. 132.

14 Michael Kasper, „Achtzehnhundertunderfroren“ - oder das Jahr ohne Sommer. Hunger, Kälte und Lawinen in den Bergregionen Westösterreichs 1816/17, in: Michael Kasper/Robert Rollinger/Andreas Rudigier (Hrsg.), Sterben in 
mehrere Vulkanausbrüche in der Nähe des Äquators, welche den Staubeintrag in die Atmosphäre immer wieder erhöhten. In Kombination mit dem Ausbruch des Vulkans Tambora im April 1815 führten diese Entwicklungen weltweit zu einer mehrjährigen Abkühlung. ${ }^{15}$ Die Temperaturen sanken dabei „um bis zu drei bis vier Grad Celsius“16.

Dass die Krise in diesen Jahren ein solch enormes Ausmaß erreichen konnte, hängt mit mehreren Faktoren zusammen. Neben den bereits geschilderten klimatischen Umständen, die vor allem von 1810 bis 1817 mehrfach zu verheerenden Missernten führten, waren es in Europa in der Folgezeit der Napoleonischen Kriege auch politische und wirtschaftliche Strukturprobleme, die Hungerkrisen in ihren extremsten AusmaBen nach sich zogen. ${ }^{17}$ Stefan Brönnimann und Daniel Krämer vom Oeschger Zentrum für Klimaforschung fassen die Situation wie folgt zusammen:

„Der Ausbruch des Tambora war aber nicht der einzige Grund für die globalen Klimaabweichungen oder die endlosen Regen in der Schweiz. Und die klimatischen Bedingungen waren nicht der einzige Grund für die geringen Ernten. Schließlich war die geringe Ernte nicht der einzige Grund für die Krise."18

Aufgrund der starken Verschränkung zwischen Klima und Gesellschaft muss auch in aller Kürze auf die schwierige wirtschaftliche und politische Lage der europäischen Gesellschaften während und nach den Napoleonischen Kriegen (1792-Juli 1815) eingegangen werden. Während der Kriegszeit brachten wechselseitige Wirtschaftsblockaden zwischen Frankreich und Großbritannien bestimmte Wirtschaftszweige ins Wanken. Allen voran geriet die Textilindustrie in der Schweiz oder auch in Vorarlberg, die von Baumwollimporten aus Großbritannien und den Kolonialgebieten abhängig war, stark unter Druck. Auch die Einfuhr von anderen Kolonialwaren (mit Ausnahme französischer) wurde durch hohen Zoll erschwert. ${ }^{19}$ Großbritannien wiederum verlor einen riesigen Absatzmarkt auf dem Kontinent, der florierende Kolonialhandel war für einige Jahre empfindlich gestört.

Gesättigte Arbeitsmärkte verstärkten die wirtschaftliche Krise. Die ehemaligen Soldaten konnten nicht alle in den Arbeitsmarkt integriert werden; des Weiteren war die Handlungsfähigkeit vieler Staaten durch die stark gestiegene Schuldenlast eingeschränkt. In der Textilindustrie kam es zudem zu einem tiefgreifenden Strukturwandel. In der Schweiz allein gingen durch die Mechanisierung von Webstühlen zwischen 1787 und 1820 etwa 60.000 Arbeitsplätze in der Handspinnerei verloren. Zahlen aus dem Kanton Zürich belegen für das Jahr 1817 bis zu 30.000 Arbeitslose im Bereich der Baumwollspinnerei. Da am Ende der Napoleonischen Kriege auch die Vorräte der meisten Men-

den Bergen. Realität - Inszenierung - Verarbeitung (Montafoner Gipfeltreffen 3/vorarlberg museum Schriften 40), Wien-Köln-Weimar 2018, S. 85-101, hier S. 86. 
schen aufgrund von Plünderungen und ständigen Einquartierungen von Soldaten zur Neige gingen, war der Weg in eine große Krise geebnet. ${ }^{20}$

Durch die quantitativ und qualitativ schlechten Ernten im „Jahr ohne Sommer" 1816 kam es zu einer Inflationskrise, bei der sich die jährlichen Durchschnittspreise zwischen 1815 und 1817 auf den europäischen Märkten verdoppelten oder sogar verdreifachten. In Rorschach (Schweiz, Kanton St. Gallen) erreichte die Teuerung sogar sechshundert Prozent. Daraus ergaben sich dramatische Folgen: Vor allem Menschen aus den Unterund Mittelschichten konnten sich Lebensmittel nicht mehr leisten, Missernten in der Landwirtschaft beraubten Knechte und Taglöhner ihrer Verdienstmöglichkeiten. Auch der Handel und das Gewerbe waren durch leere Auftragsbücher schwer betroffen. ${ }^{21}$

Sowohl klimatische als auch wirtschaftliche und politische Faktoren führten also in Europa während und nach den Napoleonischen Kriegen zu Krisenerscheinungen. Dass auch Zeitgenoss*innen schon die Folgen des kalten Klimas mit der Ereignisgeschichte in Verbindung brachten, zeigt das Beispiel des Bremer Meteorologen Wilhelm Christian Müller. Er verfasste eine Witterungsgeschichte und schrieb darin zum Winter 1812 folgende Notiz: „Vom 15. bis 24. November heftige Kälte in ganz Europa. Dadurch Vorbereitung zum Untergang Napoleons und der Franzosen in Russland."22

Obwohl somit mehrere Faktoren zu den Krisenjahren 1816 und 1817 führten, wird mit dem Ausbruch des Vulkans Tambora aber immer wieder ein einzelnes Ereignis genannt, welches Klima und Gesellschaft in dieser Zeit maßgeblich beeinflussten. ${ }^{23}$

\subsection{Der Tambora-Ausbruch und dessen Folgen}

Auf der indonesischen Insel Sumbawa brach am 5. April 1815 der Vulkan Tambora aus und schleuderte dabei in den folgenden Tagen Millionen Tonnen von Schwefel und Asche in die Erdatmosphäre. ${ }^{24}$ Der Tambora zählt zu den sogenannten Super-Vulkanen, die in der "Subduktionszone entlang der Sunda-Inseln im heutigen Indonesien"25 liegen. Weitere Vulkane in dieser Region sind unter anderem der Toba auf Sumatra, der vor etwa 75.000 Jahren ausbrach, oder der 1883 eruptierende Krakatau. Vulkanolog*innen des Massachusetts Institute of Technology (MIT) bewerteten den Tambora-Ausbruch auf dem Vulkan-Explosivitäts-Index (VEI) mit einer Stärke von sieben und damit als den größten Vulkanausbruch in den letzten 10.000 Jahren. ${ }^{26}$

20 Brönnimann/Krämer, Tambora und das "Jahr ohne Sommer", S. 28.

21 Ebd.

22 Wilhelm Christian Müller, Fünfhundertjährige Witterungs-Geschichte, besonders der außerordentlichen Kälte; nebst Beobachtungen ihrer Perioden und Einwirkungen auf die Menschheit, Bremen 1823, S. 74-75, zit. nach Wolfgang Behringer, Tambora und das Jahr ohne Sommer. Wie ein Vulkan die Welt in die Krise stürzte, München 32016, S. 33.

25 Wolfgang Behringer, Tambora und das Jahr ohne Sommer. Ein Naturereignis mit globalen Auswirkungen, in: Fabian Frommelt/Florian Hitz/Michael Kasper/Christof Töny (Hrsg.), Das Jahr ohne Sommer. Die Hungerkrise 1816/17 im mittleren Alpenraum (vorarlberg museum Schriften 31/Schriftenreihe des Arbeitskreises für interregionale Geschichte des mittleren Alpenraumes 4), Innsbruck 2017, S. 9-29, hier S. 9. 
Nachdem der Vulkan am 5. April 1815 ausgebrochen war, dauerte die erste heftige Eruptionsphase fünf Tage. Die Hauptausbruchsphase fand zwischen dem 10. und 11. April statt; während dieser beiden Tage wurden circa $50 \mathrm{~km}^{3}$ Gesteinsmaterial ausgeworfen. Die Explosionen waren zum Teil über Entfernungen von bis zu 2.600 Kilometer zu hören. Ab dem 11. April ging die Intensität des Ausbruchs zurück und dieser endete am 17. April. Dem Tambora, der zuvor der höchste Berg der Inselgruppe war, wurde im Zuge des Ausbruchs das oberste Drittel weggesprengt und er schrumpfte damit von etwa 4.350 auf 2.850 Meter Höhe. ${ }^{27}$ Neben der Eruptionssäule, die in eine Höhe von 45 Kilometer und damit bis in die Stratosphäre aufstieg, war der Ausbruch auch von Erdbeben und Tsunamis begleitet. In einem Umkreis von mehreren tausend Kilometern fiel Asche und saurer Regen. ${ }^{28}$

Was die Zahl der Todesopfer in Indonesien betrifft, herrscht in der Literatur keine Einigkeit. Oft werden etwa 100.000 Tote für das Land angegeben, ${ }^{29}$ Behringer aber kritisiert, dass dieser Wert von den meisten Autor*innen aus einem Reisebericht des Schweizers Heinrich Zollinger, der den Tambora 1847 als Erster bestieg, unreflektiert übernommen wurde. Er hält diese Zahl allein für Indonesien für viel zu gering und argumentiert, dass die weltweiten Hunger-, Seuchen- und Überschwemmungsopfer hinzugerechnet werden müssen. Das ergäbe eine Opferzahl von mehreren Hundertmillionen und damit mehr Tote als in beiden Weltkriegen zusammen. Behringer sieht allenfalls in den Pestwellen des Mittelalters einen passenden Vergleich mit den Auswirkungen der Tambora-Krise. ${ }^{30}$ Allerdings sind auch Behringers Bewertungen zu hinterfragen, da er selbst keine Quellen für diese Berechnungen angibt.

So argumentieren Brönnimann und Krämer, dass die Sommerkälte 1816 in der Schweiz nur zum Teil durch den Tambora erklärt werden könne. Der Sommer in der Gegend um Genf war um etwa zwei bis drei Grad Celsius kälter als im Zeitraum von 1799 bis 1821, der Anteil des Tambora daran sei aber gering gewesen. Durch „eine Abschätzung der strahlungsgetriebenen Abkühlung"31 lässt sich durch die Daten mehrerer mitteleuropäischer Stationen „aus der Reduktion der täglichen Temperaturspanne bei klaren Tagen"32 ein Wert von 0,6 Grad Celsius errechnen. Ein Bruchteil dieses Wertes müsse möglicherweise auch auf die reduzierte Sonnenaktivität in der Zeit des „DaltonMinimums" zwischen 1790 und 1830 zurückgeführt werden. Der Einfluss des Tambora umfasse aber nicht nur Strahlungseffekte, sondern der Ausbruch verschob möglicherweise aufgrund der ungleichen Abkühlung zwischen Land und Meer auch „die Zugbahnen der Tiefdruckgebiete über dem Nordatlantik"33.

Mitteleuropa erlebte durch die Verschiebung der Wetterlagen eine zusätzliche Abkühlung, die laut Modellsimulationen etwa 0,7 bis ein Grad Celsius betrug und direkt oder

\footnotetext{
27 Brönnimann/Krämer, Tambora und das „Jahr ohne Sommer", S. 8.

28 Behringer, Tambora und das Jahr ohne Sommer, S. 12.

29 Brönnimann/Krämer, Tambora und das "Jahr ohne Sommer", S. 28.

30 Behringer, Tambora und das Jahr ohne Sommer, S. 15.

31 Brönnimann/Krämer, Tambora und das "Jahr ohne Sommer", S. 21

32 Ebd.

33 Ebd.
} 
indirekt auf den Ausbruch des Tambora zurückgeführt werden könne. Der Rest, der bis zu drei bis vier Grad Abkühlung in Mitteleuropa betrifft, müsse durch die zufällige und interne Variabilität im Klimasystem erklärt werden. In Modellen für die Schweiz ist der größte Anteil der Abkühlung sogar gänzlich ungeklärt. Mit Sicherheit könne aber gesagt werden, dass nicht alle Vulkanausbrüche zu „Jahre[n] ohne Sommer" führten und nicht alle „Jahre ohne Sommer" auf Vulkanausbrüche zurückzuführen seien. ${ }^{34}$

Nichtsdestotrotz müssen die unmittelbaren Ereignisse und die weltweiten Folgen des Tambora-Ausbruchs genauer beleuchtet werden. Der Vulkanausbruch trug zwar vermutlich nicht die vollständige Verantwortung für die Abkühlung der Jahre 1816 und 1817, hatte jedoch einen Anteil daran. So kam es in Regionen, in denen der Ausbruch das Sonnenlicht verdunkelte, zu einer deutlichen Abkühlung. Bedingt dadurch verkürzte sich die Vegetationsperiode und gefährdete die Versorgung mit Grundnahrungsmitteln. Auch in großen Teilen der restlichen Welt kühlte es signifikant ab. Im Mai und Juni 1816 wurden beispielsweise in Nordamerika große Teile der Ernten durch drei Frostperioden vernichtet. In Indien und Südafrika führten veränderte Niederschlagsmuster 1816 durch das Ausbleiben des Monsuns zu extremer Dürre, die wiederum Viehsterben und Missernten verursachte. In Südchina und Mitteleuropa traten zum Teil extrem hohe Niederschlagsmengen auf, welche die Ernten stark beeinträchtigten. Dadurch stiegen auch Flüsse und Seen an, wie zum Beispiel der Rhein und der Bodensee in Vorarlberg, aber auch der Huang He (Gelber Fluss) und der Jangtsekiang in China. Hochwasser, Starkregen, Hagel und Stürme führten zur Beschädigung oder sogar zu Ausfällen der Ernte. Das Heu wurde ebenfalls knapp, weshalb teilweise sogar Nutztiere (Kühe, Pferde, usw.) aufgrund von Futtermangel geschlachtet werden mussten. Eine weitere Auswirkung zeigte sich in der ausbleibenden Schneeschmelze. Im Hochgebirge stauten sich große Schneemengen an, wodurch viele Gletscher rasch anschwollen. In Europa, Nordamerika, China, Indien und Südafrika gelangten Agrarprodukte (Gemüse, Getreide, Obst, Wein) in schlechter Qualität und verringerten Mengen auf den Markt, was unter anderem eine extreme Inflation der Preise hervorrief. Der Brotpreis stieg in diesen beiden Jahren teilweise auf das Zehnfache des durchschnittlichen Preises der Vorjahre an. ${ }^{35}$ Im „Jahr ohne Sommer" 1816 war aber nicht nur das Wetter extrem, sondern auch das Klima. Schlechtwettersituationen waren im Sommer 1816 nicht intensiver, sondern kamen häufiger vor. Als Beispiel dient wiederum die Schweiz. Brönnimann und Krämer halten dabei fest: „Die Niederschlagshäufigkeit war in der Schweiz stark erhöht, während die Intensität der einzelnen Regenereignisse normal war."36 Obwohl es mehr Regentage gab, galten die Tage für sich genommen als nicht ungewöhnlich. Untypisch war jedoch, dass in der Schweiz und anderen Gebieten im Alpenraum im Sommer 1816 der Schnee bis in das Tal fiel. ${ }^{37}$ Für Vorarlberg kann aufgrund der Nähe zur Ostschweiz Ähnliches beobachtet werden. Viele der zuvor beschriebenen Ereignisse und Folgen können ebenfalls in Vorarlberg in den Jahren 1816/17 gefunden werden.

34 Brönnimann/Krämer, Tambora und das "Jahr ohne Sommer", S. 21.

35 Behringer, Tambora und das Jahr ohne Sommer, S. 13-14.

36 Brönnimann/Krämer, Tambora und das "Jahr ohne Sommer", S. 20.

37 Ebd. 


\section{Hungerkrise und Naturkatastrophen: Vorarlberg in den Krisenjahren 1816/17}

\subsection{Hunger-und Versorgungskrise: Gründe und Auswirkungen}

Wie im vorhergehenden Kapitel erörtert, waren auch in Vorarlberg die Klimaschwankungen ab dem Jahr 1812 ein wesentlicher Grund für die extremen Krisenjahre 1816 und 1817. Werden die vorhandenen zeitgenössischen Berichte des Zeitraums näher betrachtet, so kann für Vorarlberg diagnostiziert werden, dass als Auswirkungen der Klimaschwankungen vor allem das wechselhafte Wetter und die erhöhten Niederschlagsmengen zum Ausmaß dieser Krise beitrugen. Aus dem Jahresbericht des Montafoner Landrichters Caspar Ratz für das Jahr 1813 geht hervor, dass im Juli wegen Unwetter und Regenfälle vor allem fruchtbarer Boden fortgespült wurde und dadurch Personen mit Grundbesitz hohe Verluste erlitten. ${ }^{38}$ In Feldkirch zerstörte Ende April 1814 ein strenger Frost die Wein- und Obstblüte und die Maisaussaat, nachdem es in den drei vorangegangenen Jahren schon Missernten gegeben hatte. ${ }^{39}$

Ein Chronist aus dem Kleinen Walsertal hielt für den Sommer 1814 große Schäden aufgrund von Überschwemmungen und Hagel fest. Im darauffolgenden Winter fielen enorme Mengen an Schnee, weshalb dieser im Februar 1815 an einigen Stellen bis zu neun Meter hoch lag und die folgenden Lawinen Schäden auf den Almen anrichteten. Der Sommer 1815 war von wechselhaftem Wetter geprägt, was bei der Heuernte große Probleme nach sich zog. Ein wesentliches Problem, das aus diesem schlechten und wechselhaften Wetter zunächst entstand, war die Futterversorgung des Viehs. Auf den Almen fanden die Tiere früh kein Futter mehr und mussten deshalb zu niedriger gelegenen Weideflächen getrieben werden. In der Folge verfügte die bäuerliche Bevölkerung über kein Heu mehr und sie konnte ihre Kühe nicht mehr durchfüttern, weshalb sie diese im Herbst noch zu verkaufen versuchten. Der Heumangel war in mehreren Gebieten Vorarlbergs (Bregenzerwald, Kleines Walsertal, Montafon) ein großes Problem. Für das Jahr 1815 wurde im Wesentlichen über verfrühte Alpabfahren, wenig Getreide, Kälte und Lawinen berichtet. Ähnliches findet sich auch in den Ostschweizer und Tiroler Quellen dieser Zeit. ${ }^{40}$

Im „Jahr ohne Sommer" 1816 und dem Hungerjahr 1817 erreichte die Krise ihren Höhepunkt. Der Chronist Daniel Müller aus dem Kleinen Walsertal fasst das Jahr 1816 wie folgt zusammen:

„Das Jahr 1816 ist ein gar spätes Jahr gewesen, es ist im Sommer u. im Herbst u. auch im Frühling gar wenig gut Wetter, wohl aber gar viel Schneewetter gewesen, dass bei Mannsgedenken kein so kurzen aber rauen Frühling, nassen u. kalten Sommer u. schlechter Herbst gewesen ist." ${ }^{\prime 41}$

38 LG Montafon, Jahresbericht 1812/13. Vorarlberger Landesarchiv, Vorlass Hubert Weitensfelder, Schachtel 2, Jahresberichte der vorarlbergerischen Landgerichte; Sutterlütti, ,Jammer, Elend und Noth!!", S. 57.

39 Christoph Volaucnik, Feldkirch in der Bayernzeit, in: Verba Volant. Onlinebeiträge des Vorarlberger Landesarchivs, Nr. 70, 24.3.2009, http://apps.vorarlberg.at/vorarlberg/pdf/vv70cvbayern.pdf, eingesehen 9.3.2020.

40 Sutterlütti, ,Jammer, Elend und Noth!!", S. 58.

41 Daniel Müller (1781-1845). Gemeindearchiv Mittelberg, Kalenderaufzeichnungen aus Mittelberg im Kleinen Walsertal, zit. nach Sutterlütti, ,Jammer, Elend und Noth!!", S. 59. 
Auch im Rheintal und am Bodensee war die Lage kritisch. Ein Chronist aus Wolfurt beschrieb das "Jahr ohne Sommer" folgendermaßen:

„1816 ist gar [ein] schlechtes Jahr, es hat gar kein Wein geben und Türgen geben, denn es hat den ganzen Sommer bereits alle Tag geregnet und im herbst früher gefrohren, der Türgen und der Wein ganz erfroren [...] so ist eine große Noht, das vihle Leuthe fast zugrunde gegangen." ${ }^{\prime 42}$

1816 war geprägt von Wetterextremen. Zwischen April und Oktober wurden insgesamt 137 Regentage gezählt. Dieser andauernde Regen führte vielerorts zu Überschwemmungen, die zusammen mit Blitzschlägen und Hagel zur Folge hatten, dass Missernten einsetzten, die wiederum Hunger und Teuerungen nach sich zogen. Schnee wurde vor allem im Sommer zum Problem, weil es regelmäßig schneite und schon ab circa achthundert Metern Seehöhe eine bis zu zwanzig Zentimeter hohe Schneedecke lag. Zwischen 1.800 und 2.300 Meter hielt sich der Schnee das ganze Jahr. ${ }^{43}$

Diese Entwicklungen fanden im „Hungerjahr"44 1817, wie es in den meisten Chroniken bezeichnet wurde, ihren Höhepunkt. Für Mensch und Vieh brach eine große Hungerkrise aus. Durch das bereits beschriebene schlechte Wetter kam es dazu, dass die bäuerliche Bevölkerung von 1811 bis 1818 weniger erntete als in den vorigen und nachfolgenden Jahren. In vielen Gebieten fielen Kartoffel-, Getreide-, Wein- und Heuernten zur Gänze aus. Vor allem der bereits angeschnittene Mangel an Heu führte dazu, dass dort, wo intensiv Viehzucht betrieben wurde (wie etwa im Bregenzerwald oder im Montafon), kaum Futter für das Vieh vorhanden war. Zeitgenössische Chroniken hoben vor allem diesen Mangel hervor, was darauf hindeuten kann, dass der Heumangel eine wesentliche Auswirkung dieser Krise war und die Menschen sehr beschäftigte. ${ }^{45}$

Ab September 1815 erreichte die Lebensmittelversorgung in Vorarlberg und Tirol einen kritischen Punkt. ${ }^{46}$ Es wurde eine Verordnung gegen den Aufkauf und die Hortung von Lebensmitteln („Hamstern”) von 1802 reaktiviert. Sie diente dem Zweck, Kreisämter und Magistrate darauf aufmerksam zu machen, dass Händler*innen und Spekulant*innen nicht das gesamte Getreide und andere Produkte aufkauften, um in Folge Preise hochzutreiben und lebensnotwendige Produkte in ausländische Gebiete zu verkaufen. Die Lage spitzte sich schnell zu. Da die Ernteaussichten nicht nur in Vorarlberg, sondern auch in den benachbarten Gebieten Bayern, Baden und Württemberg schlecht aussahen, kam es zu Preiserhöhungen, Wuchereien und Einfuhrproblemen. Gerade für Vorarlberg stellte sich dieser Umstand als problematisch heraus, da im Getreidehandel zwischen den Bodenseegebieten Vorarlberg um einiges mehr Getreide ein- als ausführte. Im August 1816 herrschte bei den Vorarlberger Verantwortlichen Klarheit, dass die Ernte nicht einmal für einen Teil des Bedarfs ausreichen würde. In

42 Wolfurter Chronist über das „Jahr ohne Sommer", o. D., zit. nach Alois Niederstätter, Geschichte Vorarlbergs. Bd. 2: Vorarlberg 1523 bis 1861. Auf dem Weg zum Land, Innsbruck 2015, S. 155.

43 Sutterlütti,,Jammer, Elend und Noth!!", S. 62-63.

44 Ebd., S. 66.

45 Ebd., S. 72

46 Ebd., S. 72. 
mehreren Schreiben richtete sich Kreishauptmann Franz Christof Daubrawa an das Präsidium in Innsbruck: Er meldete die schlechte Ernte und die prekäre Situation der Menschen, die aufgrund von drei Missernten kaum mehr Lebensmittelvorräte und Vermögen besaßen. Des Weiteren forderte der Kreishauptmann einen unverzinslichen Geldvorschuss aus Innsbruck, um überlebenswichtige Getreideeinkäufe aus den benachbarten Orten Memmingen und Überlingen zu tätigen. ${ }^{47}$

Die Innsbrucker Behörden erließen zunächst im November 1816 ein Exportverbot für Getreide und Lebensmittel. Allen voran durften Butter, Käse, Schmalz und für kurze Zeit Kartoffeln nicht ausgeführt werden. Tirol und vor allem Vorarlberg waren weiterhin vom Getreideimport aus Bayern abhängig. Da die Ernte in Bayern ebenfalls schwach ausfiel, versuchten die Verantwortlichen dort durch hohe Ausfuhrzölle die Versorgung der eigenen Bevölkerung zu sichern. Die legale Ausfuhr von Bayern nach Vorarlberg und Tirol kam deshalb im Winter 1816/17 zum Erliegen, was aber ein Aufblühen des Schmugglerwesens verursachte. Unverzolltes Getreide wurde aus Bayern von bewaffneten Banden nach Vorarlberg und Tirol geschmuggelt. ${ }^{48}$ Dadurch kam es zu teils tödlichen Auseinandersetzungen zwischen der bayerischen Mautbeamtenschaft und Schmuggelnden, die zumeist aus hungrigen Tiroler und Vorarlberger Land- und Gebirgsbewohner*innen bestanden. ${ }^{49}$ Trotzdem blieb der bayerische Markt für Vorarlbergs Korn- und Getreidehandel weiterhin geöffnet. Verhandlungen über Einfuhrerleichterungen von Getreide aus Württemberg blieben erfolglos, da die österreichische Seite keine Bereitschaft zur Aufhebung der Ausfuhrverbote für Schlachtvieh und Getreide signalisierte. ${ }^{50}$

Die Krise verschärfte sich: Die Lager waren so gut wie leer und die Preise für Lebensmittel und Getreide stiegen aufgrund der Preistreiberei stark an. Daraus resultierte eine bis dato kaum dagewesene Hunger- und Versorgungskrise. Im Winter des Jahres 1816/17 erreichten die Preise solche Auswüchse, dass beispielsweise die Vorräte an Getreide und Kartoffeln, die für die Aussaat im Frühjahr hinterlegt waren, aufgegessen wurden. Um dem Mangel an Nahrung entgegenzuwirken, mengte die hungernde Bevölkerung verschiedenste Pflanzen, Kräuter und andere unübliche Dinge in das Brot. Der Tiroler Heimatkundler Rudolf Sinwel erzählte über die Streckung des Brots Folgendes: „Das Brot wurde immer kleiner, teurer und schlechter, war schließlich nur aus Kleie gebacken und mit gehackten Brennnesseln und Heublumen, mit Erbsen, Kastanien, gemahlenen Wicken und gestoßenen Baumrinden gestreckt." ${ }^{151}$ Weitere ungewöhnliche Ernährungsgewohnheiten dieser Zeit waren laut Berichten der Verzehr von Gras, Kräutern, Katzen- und Hundefleisch. Wie dramatisch der Hunger und dessen Folgen 1817 waren, schilderte der Salzburger Mediziner Wolfgang Oberlechner:

47 Müller, Hunger in Bayern, S. 102

48 Ebd.

49 Sutterlütti, „Jammer, Elend und Noth!!", S. 72-75.

50 Ebd., S. 76

51 Rudolf Sinwel, Das Hungerjahr 1816/17, in: Tiroler Heimatblätter 16 (1938), Heft 7-8, S. 198-200, hier S. 198. 
„Die Hungersnoth ist unter allen die schrecklichste Plage der Menschen, sie übertrifft Krieg und Pest; der Hunger brachte schauderhafte Zufälle an Menschen hervor, als, stinkenden Athem, Wackeln der Zähne, unerträgliche Magenschmerzen, Faulfieber, wahre Wuth und Todh; [...] in dieser allertraurigsten Lage haben die Menschen oft zu Dingen ihre Zuflucht genommen, wodurch sie noch eher starben, zu Dingen, die nicht nährten, giftig, unverdaulich waren; auf Misthaufen gruben Kinder Knochen heraus und nagten an denselben; Gyps, Kalk, Asche, Baumrinde, Gras, Saamengehäuse verschlangen sie in der Wuth des Hungers [...]."52

Auf was für drastische Ernährungsmaßnahmen auch in Vorarlberg zurückgegriffen wurde, schildert ein Bericht des aus St. Gallen stammenden Peter Scheitlin, der ein in Vorarlberg weit verbreitetes „Brot" folgendermaßen beschreibt:

\begin{abstract}
„Man machte sich hier sogar Brot aus Obsttrester und Weintrester mit Grüsch vermischt. Ein etwa halbpfündiges Brötchen aus solchem stinkenden Obsttrester, der seit dem Herbst 1816 den ganzen Winter über im Schnee und Regen bis ins Frühjahr gelegen war [...]. Ich selbst erhielt ein solches Brötchen von daher. Kaum traute ich meinen Augen. Etwas Ungesunderes und Häßlicheres konnte nicht geessen [sic] werden. [...] Noch bewahre ich ein Stück dieses Brotes, [...] als das Traurigste aller Denkmale dieser Hungerzeit auf."53
\end{abstract}

Weitere Folgen des Hungers und der Mangelernährung waren unter anderem eine erkennbare Steigerung der Sterberate, die in Tirol und Vorarlberg 1817 in etwa bei 26 Prozent lag. ${ }^{54}$ Die mangelhafte Ernährung führte zum Teil zu Krankheiten wie Typhus („Nervenfieber“). Im Kanton St. Gallen wurden 1817 als Todesursache bei 2.000 von 8.000 Todesfällen „Auszehrung" und "Lungensucht" angegeben. Bei weiteren knapp siebenhundert Todesfällen wurde Faul-, Nerven- und Gallenfieber (Typhus) diagnostiziert. Natürlich müssen die damaligen Diagnosen aus heutiger Sicht kritisch hinterfragt werden, können aber durchaus als ein Indiz für einen Zusammenhang zwischen der durch das Klima ausgelösten Hungerkrise und der steigenden Anzahl von Toten im Jahr 1817 interpretiert werden. ${ }^{55}$

Neben der Zunahme von Krankheiten und Todesfällen nahmen Fälle von Diebstählen und Wilderei zu. Ein Jäger aus Wald am Arlberg merkte für 1817 an, dass „es noch nie so viele Wilderer gegeben habe und hauptsächlich seien es die Montafoner, die ins Klosertal herüberschlichen"56. Außerdem häuften sich vermehrt kleinkriminelle Delikte

52 Wolfgang Oberlechner, Wie kann man sich bey großer Theuerung und Hungersnoth ohne Getreid gesundes Brot verschaffen. Ein Gespräch, Salzburg 1816, S. 14-15, zit. nach Kaspar, „Achtzehnhundertundzutodegefroren”, S. 40.

53 Peter Scheitlin, Meine Armenreise in den Kanton Glarus und die Umgebungen der Stadt St. Gallen in den Jahren 1816 und 1817, nebst einer Darstellung, wie es den Armen des gesammten Vaterlandes im Jahr 1817 erging. Ein Beytrag zur Charakteristik unserer Zeit, St. Gallen 1820, S. 442-443, https://play.google.com/books/reader?id=vD 4PAAAAQAAJ\&hl=de\&pg=GBS.PP2, eingesehen 27.2.2020.

54 Kasper, "Achtzehnhundertunderfroren“, S. 90.

55 Kasper, „Achtzehnhundertundzutodegefroren“, S. 42.

56 Meinrad Tiefenthaler, Von der Jagd, Wildereien und wilden Tieren im Vorarlberger Oberland vom 16. bis zum 19. Jahrhundert, in: Jahrbuch des Vorarlberger Landesmuseumsvereins, 1941, S. 65-87, hier S. 67-68. 
wie Holz- und Fruchtdiebstahl. Gerichtsurteile dieser Zeit verdeutlichen, dass viele kleine Diebstähle mit einer Haftstrafe geahndet wurden. Mundraub galt in diesen Jahren nicht als Kavaliersdelikt. Ein Beispiel aus dem Gericht Dornbirn zeigt, wie mit solchen Delikten umgegangen wurde: „Feldfrüchtediebstahl: Katharina Fröwis erhielt in Hohenems 12 Tage Arrest. Magdalena Rein in Dornbirn 6 Tage und Joseph Schwendinger, Bauer von Dornbirn, 10 Tage einfachen Arrest." ${ }^{57}$ Ein weiteres Problem stellte die stark steigende Armut in diesem Zeitraum dar, was in Vorarlberg vor allem anhand der intensiven Auseinandersetzung der Behörden mit der Bettlerproblematik ersichtlich wird. ${ }^{58}$ Erst ab 1818 verbesserte sich die Situation in Vorarlberg allmählich wieder. Ein wesentlicher Faktor, der zu dieser Hungerkrise führte, waren die Naturkatastrophen.

\subsection{Naturkatastrophen: Lawinen und Überschwemmungen}

Im südlichen Teil Vorarlbergs trugen der starke Schneefall und die daraus resultierenden Lawinen zur Krise bei. Zwischen Herbst 1815 und Sommer 1817 akkumulierten sich im Hochgebirge enorme Schneemengen. Die Kombination des schneereichen Winters 1815/16 mit einem feucht-kühlen Sommer 1816 und dem darauffolgenden schneereichen Winter 1816/17 führte dazu, dass im anschließenden Frühjahr ungewöhnlich große Mengen an Schnee in weiten Teilen des Alpenraumes lagen. In zahlreichen zeitgenössischen Berichten sind Schneerekorde greifbar - so auch in Vorarlberg. In St. Gallenkirch betrug die Schneehöhe am 9. März 1817 etwa 3,5 Meter. Im Silbertal (Montafon) war im März 1817 im Tal der Schnee 1,5 Meter hoch. Die Bewohner*innen von Bartholomäberg konnten den Quellen zufolge im Mai nur mit Brettern an ihren Füßen außer Haus gehen. ${ }^{59}$ So kam es von März bis April 1817 wegen der hohen Niederschlagsmengen in Vorarlberg wie auch in anderen alpinen Regionen zu mehreren folgenschweren Lawinenabgängen. Im Kleinen Walsertal richtete eine Lawine am 9. Jänner 1816 großen Schaden an. ${ }^{60}$ Am 9. März 1817 forderte ein Lawinenabgang in St. Gallenkirch mehrere Todesopfer. ${ }^{61}$ Weitere Berichte über Lawinenabgänge stammen unter anderem aus Gaschurn und erneut aus St. Gallenkirch am 1. Mai. Bei diesen entstanden zwar schwere Sachschäden, jedoch kamen keine Menschen ums Leben. ${ }^{62}$ Obwohl Lawinen in diesen beiden Krisenjahren einige Todesopfer forderten und gröBere Schäden anrichteten, müssen diese im gesamten Alpenraum aber als ein immer wieder vorkommendes Naturereignis betrachtet werden. Auffallend und unnatürlich ist jedoch, dass diese zum Teil noch im Frühling auftraten.

Die Überschwemmungen des Jahres 1817 nahmen jedoch ein bis zur heutigen Zeit nicht mehr erreichtes Ausmaß an. Schon 1816 führte der andauernde Regen zu Überschwemmungen des Bodensees und des Rheins. In Hard, Fußach und Vorkloster (heu-

57 Sutterlütti, ,Jammer, Elend und Noth!!“, S. 91.

58 Ebd., S. 104-105.

59 Kasper, „Achtzehnhundertunderfroren“, S. 94.

60 Sutterlütti, ,Jammer, Elend und Noth!!", S. 58.

61 Für einen besseren Eindruck siehe den Augenzeugenbericht des Bartholomä Marlin in: Inge Bacher, Aus dem Tagebuch des Bartholomä Marlin (1801-1878), in: Bludenzer Geschichtsblätter (2003), Heft 69, S. 14-17.

62 Kasper, ${ }^{\prime \prime}$ Achtzehnhundertundzutodegefroren", S. 30. 
tiger Stadtteil von Bregenz) ging der Bodensee über die Ufer. In Fußach hatte der See fast ein Drittel der für die Bewohner*innen wichtigen Wiesen unter Wasser gesetzt. ${ }^{63}$ In seiner Pfarrchronik berichtete der Fußacher und ab 1819 Harder Pfarrer Gallus Hasler über die Auswirkungen in Hard: „den 20. July fing es an zu regnen und so bis 31. Dieß, wo der See so groß wurde, daß bereits das ganze Dorf [Hard] mit samt den Feldern unter Wasser gesetzt, und sehr großen Schaden gethan hat." ${ }^{\prime 64}$ Dies sollte aber erst der Vorläufer für das bisher größte Bodensee-Hochwasser im Jahr 1817 sein.

Im Juli 1817 erreichte der Bodensee als Spätfolge des „Jahres ohne Sommer" seinen bisher höchsten bekannten Stand seit 1566. Dieser war 2,26 Meter über dem durchschnittlichen Wasserstand im Sommer. Zustande kam dieser Hochstand durch die auBergewöhnlichen meteorologischen Bedingungen, die sich in den zwanzig Monaten zuvor abgespielt hatten. Beginnend mit dem schon erwähnten schneereichen Winter 1815/16 folgte das "Jahr ohne Sommer" 1816, in dem es in den Sommermonaten bis in die Täler hinunter schneite. Im Gebirge fiel in der Sommerzeit weiterer Schnee. Durch den Winter 1816/17 und das kalt-feuchte Frühjahr 1817 legte sich über dieses bereits doppelte Schneepaket noch ein drittes. Die hochliegende Nullgradgrenze durch die für mehrere Wochen sommerliche Witterung und häufige Wärmegewitter führten dazu, dass um den Juni 1817 die ungeheuren Schneemassen rasch schmolzen - dieses Phänomen wird als doppelte Schneeschmelze bezeichnet. Der Schnee aus dem Winter 1815/16 schmolz gleichzeitig mit dem der Winterperiode 1816/17 und führte deshalb dazu, dass der Bodensee eine Rekordhöhe erreichte, die etwa 89 Tage andauerte. ${ }^{65}$

Fast das gesamte Rheintal war über Monate hinweg überflutetet, was katastrophale Auswirkungen für die Ernte hatte und in zehn Dörfern fast alle Felder und Früchte betraf. Ihren Höhepunkt erreichte die Überschwemmung im Juli 1817. In Lustenau etwa ging der Rhein über. Durch gemeinsame Anstrengung der Einheimischen konnte das Schlimmste verhindert werden. Für die direkt am Bodensee gelegenen Gemeinden, wie zum Beispiel Bregenz oder Hard, war die Lage jedoch dramatisch. Am 7. Juli 1817 erreichte der Bodensee seinen historischen Höchstwert. Der Kornmarkt in Bregenz und Teile von Vorkloster standen unter Wasser. Kreishauptmann Daubrawa berichtete am 15. Juli nach Innsbruck wie folgt:

"Auch hier in der Stadt Bregenz, worin man mit Schiffen fahren kann, wurde gestern von einer eben derselben Woge eine sehr feste und hohe Gartenwehr und zugleich ein weit dahinter gestandenes Gartenhäuschen mit einem Wurfe umgeworfen. Die Verheerungen des wüthenden Sees (der auf der benachbarten Stadt Lindau die ganze Brücke wegzureißen droht) sind höchst schauderhaft, und zugleich von den Ortschaften Hard, Fußach und Gaißau einlangende Nachrichten lauten auf das Kläglichste, und nehmen eine fortwährende ergie-

63 Sutterlütti, „Jammer, Elend und Noth!!”, S. 60

64 Hasler Chronik 1816. Gemeindearchiv Hard, II. 1 Pfarrbeschreibung, zit. nach Sutterlütti, „Jammer, Elend und Noth!!", S. 60.

65 Christian Pfister, Wetternachhersage. 500 Jahre Klimavariationen und Naturkatastrophen (1496-1995), Bern 1999, S. 228-229. 
bige Unterstützung der von allem entblößten Einwohner mit Lebensmitteln gebietherisch in Anspruch, um ihnen wenigstens die kümmerlichste Hilfe zu leisten." ${ }^{66}$

Die „Linzer Zeitung“ berichtete am 18. Juli über die Lage zu Beginn des Monats: ${ }^{67}$

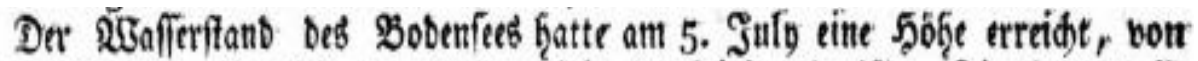

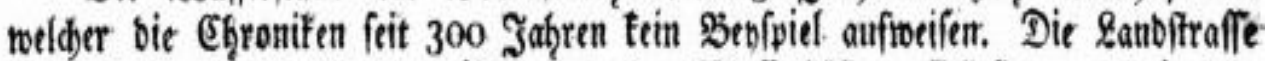

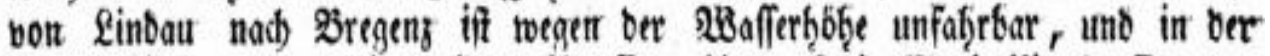

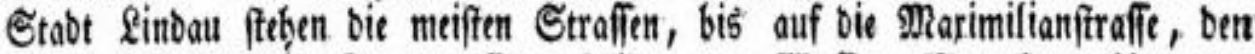

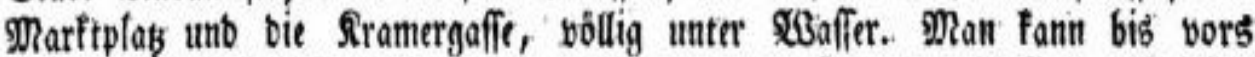

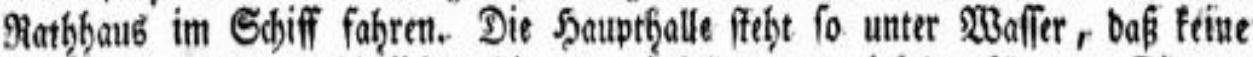

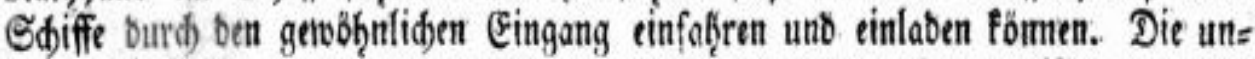

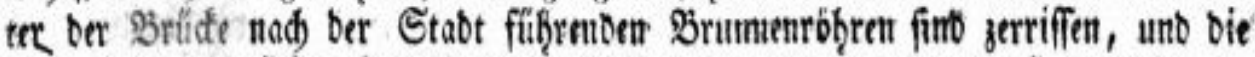

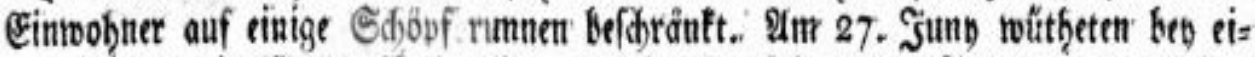
nemt Sturm bie Wellen fürdfertidfy, une braden fidy ant Der Etabt und ben llfertr

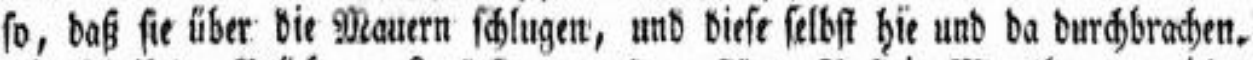

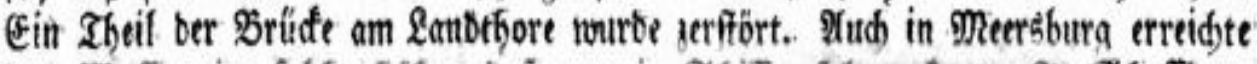

Auch die „Klagenfurter Zeitung" berichtete am 30. Juli 1817 über die missliche Lage der Bodensee-Ortschaften: ${ }^{68}$

$$
B \circ \text { r a r I berg. }
$$

Sirtgens vom 19. Suti.

Dab ginfitige Metter, melder feit bem 3. Tat aud in Borar!berg zum Blíc Des gangen Eandes nad vier pifijabren eintrat,

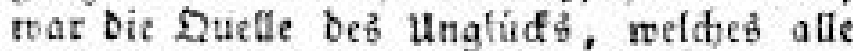
an bem Bobeniee getegenen Drtídhaften traf.

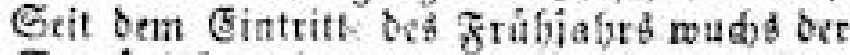
Gec táglid mefre an, unb uberftieg an 6.

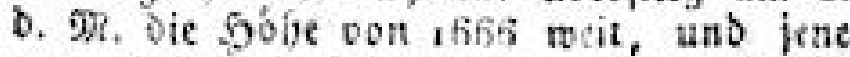
oon 6560 um einige 3olte. Die obnebin 
Die Lage in Vorarlberg besserte sich zwar nach dem Sommer 1817, war aber zweigeteilt. Die mittleren und höheren Gebiete wie der Bregenzerwald, das Walgau und das Montafon konnten wieder Ernteerträge wie in Vorkrisenzeiten einfahren. Im Rheintal und allen voran am Bodensee blieb die Situation aber noch einige Monate kritisch, da die Ernte erneut vernichtet wurde. Die Totalausfälle hatten zur Folge, dass trotz der Besserung der allgemeinen Situation viele Menschen im Winter 1817/18 und dem darauffolgenden Frühjahr mit dem Nötigsten versorgt werden mussten. Für Tirol und Vorarlberg waren in dieser Übergangszeit vor allem Getreideimporte aus der Lombardei von großer Bedeutung. Jedoch wurde die Stadt Bregenz im Herbst 1817 mit ausreichend Vorräten ausgestattet, womit die Nachbargemeinden Vorkloster und Hard beliefert werden konnten. ${ }^{69}$ Mit dem Jahr 1818 verbesserte sich die Lage, im kollektiven Gedächtnis der Vorarlberger Bevölkerung waren jene Krisenjahre aber noch über Generationen hinweg tief verankert und wurden vor allem durch mündliche Geschichten an die jüngeren Generationen weitergetragen. ${ }^{70}$

\section{Fazit}

Ausgangspunkt der Arbeit waren die Fragen, ob das "Jahr ohne Sommer" hauptursächlich auf den Tambora-Ausbruch zurückzuführen ist und wie sich die Krise auf die Vorarlberger Bevölkerung auswirkte. Eruiert wurde dabei, dass die Krisenjahre 1816 und 1817 in Vorarlberg viele unterschiedliche Gründe hatten und nicht auf den Ausbruch des Tambora allein zurückgeführt werden können. Der Begriff der „Tamborakrise“, wie er von Wolfgang Behringer geprägt wurde, ist somit nicht zielführend. Selten sind es Einzelereignisse, die unvermittelt eine solche Katastrophenlage auslösen. Meistens stehen verschiedene Gründe und Entwicklungen dahinter, die teilweise Jahre, Jahrzehnte, oder bei Klimaschwankungen sogar Jahrhunderte im Gange waren. Ähnliches ist auch für die Jahre 1816/17 in Vorarlberg zu beobachten. Als klimatische Faktoren sind zum einen eine Kälteperiode in den 1810 er-Jahren zu nennen, die auch als Höhepunkt der "Kleinen Eiszeit" bezeichnet wird und schon in den Jahren vor dem Tambora-Ausbruch ihre Auswirkungen zeigte. Zum anderen kam es zu einer geringeren Sonnenaktivität in der Zeit des sogenannten "Dalton-Minimums", die zusammen mit der ozeanischen Variabilität dazu führte, dass in diesem Zeitraum die Durchschnittstemperatur im Vergleich mit dem 20. Jahrhundert in vielen Gebieten Europas um drei bis vier Grad Celsius absank. Es waren aber nicht nur die klimatischen Schwankungen, die fast ganz Europa in eine Krise stürzten. Aus den Napoleonischen Kriegen resultierten ausgeprägte Wirtschaftskrisen, die maßgeblich zur Hungerkrise beitrugen.

In Vorarlberg waren die Auswirkungen des Zusammenspiels dieser unterschiedlichen Faktoren verheerend. Zu der schon angespannten wirtschaftlichen Situation kamen noch das schwankende Klima und dessen Folgen dazu. Dies führte unter anderem dazu, dass die Niederschlagstage mehr wurden und in höheren Lagen (ab 1.800 Meter)

69 Sutterlütti, ,Jammer, Elend und Noth!!”, S. 81-82.

70 Kasper, ${ }_{1,}$ Achtzehnhundertundzutodegefroren", S. 11. 
das ganze Jahr über Schnee lag. Für die Ernte waren die Auswirkungen dieser klimatischen Umstände fatal. Schnee, Hagel, Stürme, Überschwemmungen und Lawinen zerstörten spätestens ab 1816 in großen Teilen Vorarlbergs die Ernten und führten zu einer kaum dagewesenen Hunger- und Versorgungskrise. Daraus folgte eine Mangelernährung der Bevölkerung mit erhöhtem Aufkommen von Krankheiten und Sterbefällen. Da auch die Armut sehr hoch war, nahmen Fälle von Bettelei, Diebstählen oder Wilderei zu.

Die Situation besserte sich ab 1818 wieder, jedoch blieben in der Vorarlberger Bevölkerung über mehrere Generationen die Erfahrungen dieser harten Zeit tief im kollektiven Gedächtnis verankert. Berichte von Zeitzeug*innen belegen, wie Geschichten über diese Zeit an die nächsten Generationen weitergetragen wurden. Abschließend soll festgehalten werden, dass das Einbeziehen von klimageschichtlichen Methoden und Erklärungsversuchen ein Gewinn für die Geschichtswissenschaft sein kann. Jedoch sollte auch in diesem Zusammenhang nicht der Fehler begangen werden, einzelnen Naturkatastrophen (wie eben einem Vulkanausbruch) eine singuläre Bedeutung beizumessen.

\section{Quellen}

Faszikel D. D. über die bezirksweise Noth wegen Überschwemmung, Daubrawa, Bregenz, 15. Juli 1817. Vorarlberger Landesarchiv, Karton 1, Schachtel 308.

Hasler Chronik 1816. Gemeindearchiv Hard, II. 1 Pfarrbeschreibung.

Klagenfurter Zeitung, 30.7.1817.

Linzer Zeitung, 18.7.1817.

LG Montafon, Jahresbericht 1812/13. Vorarlberger Landesarchiv, Vorlass Hubert Weitensfelder, Schachtel 2, Jahresberichte der vorarlbergerischen Landgerichte.

Müller, Daniel (1781-1845). Gemeindearchiv Mittelberg, Kalenderaufzeichnungen aus Mittelberg im Kleinen Walsertal.

Oberlechner, Wolfgang, Wie kann man sich bey großer Theuerung und Hungersnoth ohne Getreid gesundes Brot verschaffen. Ein Gespräch, Salzburg 1816.

Scheitlin, Peter, Meine Armenreise in den Kanton Glarus und die Umgebungen der Stadt St. Gallen in den Jahren 1816 und 1817, nebst einer Darstellung, wie es den Armen des gesammten Vaterlandes im Jahr 1817 erging. Ein Beytrag zur Charakteristik unserer Zeit, St. Gallen 1820, https://play.google.com/books/reader?id=vD4PAAAAQA AJ\&hl=de\&pg=GBS.PP2, eingesehen 27.2.2020. 


\section{Literatur}

Bacher, Inge, Aus dem Tagebuch des Bartholomä Marlin (1801-1878), in: Bludenzer Geschichtsblätter (2003), Heft 69, S. 14-17.

Behringer, Wolfgang, Tambora und das Jahr ohne Sommer. Wie ein Vulkan die Welt in die Krise stürzte, München 2016.

Ders., Tambora und das Jahr ohne Sommer. Ein Naturereignis mit globalen Auswirkungen, in: Fabian Frommelt/Florian Hitz/Michael Kasper/Christof Töny (Hrsg.), Das Jahr ohne Sommer. Die Hungerkrise 1816/17 im mittleren Alpenraum (vorarlberg museum Schriften 31/Schriftenreihe des Arbeitskreises für interregionale Geschichte des mittleren Alpenraumes 4), Innsbruck 2017, S. 9-29.

Brönnimann, Stefan/Krämer, Daniel, Tambora und das „Jahr ohne Sommer" 1816. Klima, Mensch und Gesellschaft (Geographica Bernensia G90), Bern 2016.

Fankhauser, Andreas, Kontinentalsperre, in: Historisches Lexikon der Schweiz (HLS), 30.10.2008, https://hls-dhs-dss.ch/de/articles/013928/2008-10-30/, eingesehen 25.2.2021.

Kasper, Michael, "Achtzehnhundertundzutodegefroren“, in: Edith Hessenberger/Michael Kasper/Andreas Rudigier/Bruno Winkler (Hrsg.), Jahre der Heimsuchung. Historische Erzählbilder von Zerstörung und Not im Montafon (Sonderband zur Montafoner Schriftenreihe 12), Schruns 2010, S. 9-71.

Ders., ,Achtzehnhundertunderfroren“ - oder das Jahr ohne Sommer. Hunger, Kälte und Lawinen in den Bergregionen Westösterreichs 1816/17, in: Michael Kasper/Robert Rollinger/Andreas Rudigier (Hrsg.), Sterben in den Bergen. Realität - Inszenierung - Verarbeitung (Montafoner Gipfeltreffen 3/vorarlberg museum Schriften 40), Wien-KölnWeimar 2018, S. 85-101.

Müller, Christian Wilhelm, Fünfhundertjährige Witterungs-Geschichte, besonders der außerordentlichen Kälte; nebst Beobachtungen ihrer Perioden und Einwirkungen auf die Menschheit, Bremen 1823.

Müller, Gerald, Hunger in Bayern 1816-1818. Politik und Gesellschaft in einer Staatskrise des frühen 19. Jahrhunderts (Europäische Hochschulschriften III, Geschichte und ihre Hilfswissenschaften 812), Frankfurt am Main u. a. 1998.

Niederstätter, Alois, Geschichte Vorarlbergs, Bd. 2: Vorarlberg 1523 bis 1861. Auf dem Weg zum Land, Innsbruck 2015.

Pfister, Christian, Klimageschichte der Schweiz 1525-1860. Das Klima der Schweiz von 1525-1860 und seine Bedeutung in der Geschichte von Bevölkerung und Landwirtschaft, Bd. 1 (Academica helvetica 6), Bern-Stuttgart ${ }^{2} 1985$.

Ders., Wetternachhersage. 500 Jahre Klimavariationen und Naturkatastrophen (14961995), Bern 1999. 
Sinwel, Rudolf, Das Hungerjahr 1816/17, in: Tiroler Heimatblätter 16 (1938), Heft 7-8, S. 198-200.

Sutterlütti, Sabine, „Jammer, Elend und Noth!!”. Vorarlberger 1814 bis 1818 (Institut für sozialwissenschaftliche Regionalforschung Veröffentlichungen 14), Regensburg 2016.

Tiefenthaler, Meinrad, Von der Jagd, Wildereien und wilden Tieren im Vorarlberger Oberland vom 16. bis zum 19. Jahrhundert, in: Jahrbuch des Vorarlberger Landesmuseumsvereins, 1941, S. 65-87.

Volaucnik, Christoph, Feldkirch in der Bayernzeit, in: Verba Volant. Onlinebeiträge des Vorarlberger Landesarchivs, Nr. 70, 24.3.2009, http://apps.vorarlberg.at/vorarlberg/pdf/ vv70cvbayern.pdf, eingesehen 9.3.2020.

\section{Abbildungen}

Abbildung 1: Linzer Zeitung vom 18.7.1817, S. 4.

Abbildung 2: Klagenfurter Zeitung vom 30.7.1817, S. 2-3.

Florian Guggenberger ist Student des Masterstudiums Geschichte an der Universität Innsbruck und Mitarbeiter des Stadtarchivs Bregenz. Florian.Guggenberger@student. uibk.ac.at.

\section{Zitation dieses Beitrages}

Florian Guggenberger, Vorarlberg in den Krisenjahren 1816 und 1817: Gründe und Auswirkungen, in: historia.scribere 13 (2021), S. 127-145, [http://historia.scribere.at], eingesehen 22.6.2021 (=aktuelles Datum).

Creative Commons Licences 3.0 Österreich unter Wahrung der Urheberrechte der Autorlnnen. 
